\title{
SIGNAL SUBSPACE TRANSFORMATION FOR DIRECTION-OF-ARRIVAL ESTIMATION OF WIDEBAND SOURCES IN NEAR FIELD
}

Lena Chang

Department of Communications, Navigation and Control Engineering, National Taiwan Ocean University, Taiwan, R.O.C, lenachang@mail.ntou.edu.tw

Follow this and additional works at: https://jmstt.ntou.edu.tw/journal

Part of the Electrical and Computer Engineering Commons

\section{Recommended Citation}

Chang, Lena (2010) "SIGNAL SUBSPACE TRANSFORMATION FOR DIRECTION-OF-ARRIVAL ESTIMATION OF WIDEBAND SOURCES IN NEAR FIELD," Journal of Marine Science and Technology. Vol. 18: Iss. 6, Article 7. DOI: 10.51400/2709-6998.1941

Available at: https://jmstt.ntou.edu.tw/journal/vol18/iss6/7

This Research Article is brought to you for free and open access by Journal of Marine Science and Technology. It has been accepted for inclusion in Journal of Marine Science and Technology by an authorized editor of Journal of Marine Science and Technology. 


\title{
SIGNAL SUBSPACE TRANSFORMATION FOR DIRECTION-OF-ARRIVAL ESTIMATION OF WIDEBAND SOURCES IN NEAR FIELD
}

\author{
Lena Chang*
}

Key words: signal subspace transformation, tracking, direction-ofarrival (DOA) estimation, near-field, wideband sources.

\begin{abstract}
A novel adaptive signal subspace transformation for direction-of-arrival (DOA) estimation of wideband sources in the near-field is proposed in this paper. The method is composed of two transformations: signal subspace focusing (SSF) and far-field transformation (FFT). SSF aligns the signal subspaces for different frequencies in the bandwidth of the sources based on focusing matrices. FFT transforms the near-field steering vector to be the far-field steering vector with same direction-of-arrival (DOA) allocation. By the way, we simplify the location estimation of wideband sources in the near-field to be narrowband sources in the far field. The proposed method can track the DOAs and ranges of near-field wideband sources, simultaneously. Simulation results and water-tank experiments confirm the feasibility and efficiency of the proposed adaptive DOA estimation method in underwater environments.
\end{abstract}

\section{INTRODUCTION}

The estimation of direction-of-arrival (DOA) has been widely applied in the field of radar, sonar, communications and seismology. Most of DOA estimation algorithms are restricted to the narrowband sources in far-field. Recently, there has been growing interest in developing wideband source location estimators in the near-field, in which both the angles and the ranges are required to be estimated simultaneously. In this study, we present an efficient adaptive DOA estimator based on the recursive signal subspace transformation for wideband source estimation and tracking.

Two-dimension (2-D) MUSIC method and the maximum likelihood estimator (MLE) [3] determine the range and the angle of sources, which can be considered to be the modified versions of far-field cases. Some other methods based on the

Paper submitted 07/31/09; revised 05/06/10; accepted 09/10/10. Author for correspondence: Lena Chang (e-mail: lenachang@mail.ntou.edu.tw).

*Department of Communications, Navigation and Control Engineering, National Taiwan Ocean University, Taiwan, R.O.C. second-order statistics have been proposed in the literature, such as the weighted linear prediction method [2], and the generalized ESPRIT method [12]. [8] introduced an underwater acoustic eigenstructure ESPRIT-based algorithm which derived closed-form DOA estimates for near-field sources by using a single vector hydrophone. To overcome the multipath underwater acoustic problem, [7] proposed a non-iteration deconvolution algorithm for shallow water multipath channel estimation in near range. [5] developed a far-field approximation (FFA) method which constructs a FFA covariance matrix from the measured covariance matrix to approximate the ideal covariance matrix associated with the far-field signals whose bearings are the same as those of the near-field signals. Recently, [11] estimated the DOA and range of nearfield sources based on joint diagonalization, which obtained the azimuth and range parameters without 2-D parameter search. These methods deal with the narrowband assumption.

Furthermore, [4] proposed the coherent signal-subspace method (CSM) for the direction estimation of wideband sources. Although the CSM is very effective in the wideband signal detection and estimation, it suffers from the construction of the focusing matrices which require a set of preliminary DOA estimates via low resolution methods, such as beamforming or spectrum paradigm. If these preliminary DOA estimates are inappropriately selected, the CSM often results in an estimation error in the focusing matrices. [10] transformed the received data to the frequency domain, and each frequency bin can be represented by the narrowband model. These methods are proposed for the estimation of far-field wideband sources.

In the study, we propose an estimator to solve the problem of joint range and DOA estimation for multiple wideband near field sources. To alleviate the performance degradation caused by wideband and near-field sources, we develop an adaptive DOA estimator which combines signal subspace focusing (SSF) and far-field transformation (FFT) based on the adaptive eigen-decomposition algorithm [1]. We first estimate the signal subspace corresponding to each frequency bin of the received wideband signals by using an adaptive eigen-decomposition algorithm, which can calculate the eigencomponents of the signal subspace in real time. Then, the FFT is applied to near-field direction and range estimation. The proposed FFT utilizes a far-field transformation matrix 
to transform the near-field steering vector into the far-field steering vector with same DOA allocation. By using the FFT, we simplify the near-field DOA estimation as the far-field estimation. Finally, we propose a SSF method for the DOAs estimation of wideband sources. The SSF transforms the signal subspace, which corresponds to the spectrum density matrix, at each frequency bin onto the focusing subspace (usually the signal subspace at receiver center frequency) by using focusing matrices. The focusing matrix is formed without a priori knowledge of source angles. Thus, the SSF avoids the sensitivity problem caused by preliminary angle estimate. The proposed method can track the DOAs and ranges of the wideband sources in near-field, simultaneously.

\section{PROBLEM FORMULATION}

Consider a $p$-element linear array illuminated by $q$ nearfield wideband sources. The source signals are limited to a common frequency band with bandwidth $\mathrm{B}$ and center frequency $f_{o}$. The signal received at the $m$ th sensor can be expressed as

$$
x_{m}(t)=\sum_{i=1}^{q} \alpha_{i m} s_{i}\left(t-\tau_{i m}\right)+n_{m}(t)
$$

where $\alpha_{i m}$ is the complex gain of the mth sensor with respect to the $i$ th source. $\tau_{i m}$ is the propagation delay for the $i$ th source at the sensor $m$ with respect to the reference point of the array. $s_{i}(t)$ is the amplitude of $i$ th source and $n_{m}(t)$ is the additive noise at the sensor $m$. The received signal $x_{m}(t)$ can be represented by its Fourier components $X_{m}\left(f_{k}\right)$ in the frequency domain. The k-th Fourier components corresponding to the array measurements $x_{m}(t), m=1, \ldots, p$, can be expressed as a vector form,

$$
\mathbf{X}\left(f_{k}\right)=\left[\begin{array}{c}
X_{1}\left(f_{k}\right) \\
X_{2}\left(f_{k}\right) \\
\vdots \\
X_{P}\left(f_{k}\right)
\end{array}\right]=\mathbf{A}\left(\theta, d, f_{k}\right) \mathbf{S}\left(f_{k}\right)+\mathbf{N}\left(f_{k}\right)
$$

where $\mathbf{S}\left(f_{k}\right)=\left[S_{1}\left(f_{k}\right), S_{2}\left(f_{k}\right), S_{3}\left(f_{k}\right) \ldots S_{q}\left(f_{k}\right)\right]^{T}$ and $\mathbf{N}\left(f_{k}\right)=$ $\left[N_{1}\left(f_{k}\right), N_{2}\left(f_{k}\right), N_{3}\left(f_{k}\right) \ldots N_{P}\left(f_{k}\right)\right]^{T} . \mathbf{S}\left(f_{k}\right)$ and $\mathbf{N}\left(f_{k}\right)$ denote the vectors formed by the Fourier components of signal and noise at frequency $f_{k}$, respectively. $\mathbf{A}\left(\theta, d, f_{k}\right)$ is a $p \times q$ matrix and represents the array direction matrix corresponding to frequency $f_{k}$, given by

$$
\mathbf{A}\left(\theta, d, f_{k}\right)=\left[a\left(\theta_{1}, d_{1}, f_{k}\right) \quad a\left(\theta_{2}, d_{2}, f_{k}\right) \quad \ldots . \quad a\left(\theta_{q}, d_{q}, f_{k}\right)\right]
$$

In (3), $a\left(\theta_{i}, d_{i}, f_{k}\right)$ represents the near-field direction vector of the $i$ th source located at direction $\theta_{i}$ and range $d_{i}$ at frequency $f_{k}$, $a\left(\theta_{i}, d_{i}, f_{k}\right)=\left[\alpha_{i 1} e^{-j 2 \pi f_{k} \tau_{i 1}}, \alpha_{i 2} e^{-j 2 \pi f_{k} \tau_{i 2}}, \ldots, \alpha_{i p} e^{-j 2 \pi f_{k} \tau_{i p}}\right]^{T}$

and $\tau_{i m}=\left[\sqrt{\left(d_{i}-y_{m} \sin \theta_{i}\right)^{2}+\left(y_{m} \cos \theta_{i}\right)^{2}}-d_{i}\right] / c$, where $y_{m}$ is the $m$ th sensor location and $c$ is the propagation velocity. Suppose the noise $\mathbf{N}\left(f_{k}\right)$ is independent of the signal $\mathbf{S}\left(f_{k}\right)$ and has zero mean with known noise spectrum density matrix $\mathbf{P}_{N}\left(f_{k}\right)=E\left[\mathbf{N}\left(f_{k}\right) \mathbf{N}^{H}\left(f_{k}\right)\right]=\sigma^{2} \mathbf{P}_{\eta}\left(f_{k}\right)$. The spectrum density matrix of the array output is given by

$$
\begin{aligned}
\mathbf{P}_{X}\left(f_{k}\right) & =E\left[\mathbf{X}\left(f_{k}\right) \mathbf{X}^{H}\left(f_{k}\right)\right] \\
& =\mathbf{A}\left(\theta, d, f_{k}\right) \mathbf{P}_{s}\left(f_{k}\right) \mathbf{A}\left(\theta, d, f_{k}\right)^{H}+\mathbf{P}_{N}\left(f_{k}\right)
\end{aligned}
$$

where $\mathbf{P}_{s}\left(f_{k}\right)=E\left[\mathbf{S}\left(f_{k}\right) \mathbf{S}\left(f_{k}\right)^{H}\right]$ is the source spectrum density matrix and $\mathbf{P}_{N}\left(f_{k}\right)=\sigma^{2} I$ for white noise.

\section{FAR-FIELD TRANSFORMATION (FFT)}

In the section, we propose the FFT to transform the nearfield direction vector $a\left(\theta_{i}, d_{i}, f_{k}\right)$ to be far field with the same signal direction. In (4), $a\left(\theta_{i}, d_{i}, f_{k}\right)$ can be approximated to be

$$
a\left(\theta_{i}, d_{i}, f_{k}\right) \approx Q\left(\theta_{i}, d_{i}, f_{k}\right) a\left(\theta_{i}, f_{k}\right)
$$

where $a\left(\theta_{i}, f_{k}\right)$ is the far-field steering vector of source located at $\theta_{i}$,

$$
a\left(\theta_{i}, f_{k}\right)=\left[e^{j 2 \pi f_{k} y_{1} \sin \theta_{i} / c}, e^{j 2 \pi f_{k} y_{2} \sin \theta_{i} / c}, \ldots, e^{j 2 \pi f_{k} y_{P} \sin \theta_{i} / c}\right]^{T}
$$

and $Q\left(\theta_{i}, d_{i}, f_{k}\right)$ represents the transformation between the farfield and near-field steering vectors,

$$
\begin{gathered}
Q\left(\theta_{i}, d_{i}, f_{k}\right)=\operatorname{diag}\left(\begin{array}{llll}
q_{i 1} & q_{i 2} & \ldots & q_{i p}
\end{array}\right) \\
\text { with } q_{i m}=\alpha_{i m} e^{-j 2 \pi f_{k}\left(y_{m} \cos \theta_{i}\right)^{2} /\left(2 d_{i} c\right)}
\end{gathered}
$$

in 2D-MUSIC, the steering vector $a\left(\theta, d, f_{k}\right)$ is projected onto the noise space $E_{n}\left(f_{k}\right)$ and the parametric search in angle and range revealing the true directional vectors as the spectrum $p(\theta$, $d, f_{k}$ ) achieves the null values. The null spectrum is defined as

$$
\begin{aligned}
p\left(\theta, d, f_{k}\right) & =a^{H}\left(\theta, d, f_{k}\right) E_{n}\left(f_{k}\right) E_{n}^{H}\left(f_{k}\right) a\left(\theta, d, f_{k}\right) \\
& =a^{H}\left(\theta, d, f_{k}\right)\left[I-E_{s}\left(f_{k}\right) E_{s}^{H}\left(f_{k}\right)\right] a\left(\theta, d, f_{k}\right)
\end{aligned}
$$

$E_{s}\left(f_{k}\right)$ and $E_{n}\left(f_{k}\right)$ are the signal and noise subspace corresponding to spectrum density matrix of the array output $\mathbf{P}_{X}\left(f_{k}\right)$. Substituting (6) into (9), the null spectrum becomes 


$$
p\left(\theta, f_{k}\right)=a^{H}\left(\theta, f_{k}\right)\left[I-E_{s}^{\prime}\left(f_{k}\right) E_{s}^{\prime H}\left(f_{k}\right)\right] a\left(\theta, f_{k}\right)
$$

where $E_{s}^{\prime}\left(f_{k}\right)=Q^{H}\left(\theta, d, f_{k}\right) E_{s}\left(f_{k}\right)$. From (10), we know that the two-dimension parametric search in angle $\theta$ and range $d$ can be reduced to one-dimension angle search, as the signal subspace $E_{s}\left(f_{k}\right)$ corresponding to the near-field sources is transformed to be the associated far-field signal subspace $E_{s}^{\prime}\left(f_{k}\right)$. However, the transformation matrix $Q\left(\theta, d, f_{k}\right)$ is required to construct $E_{s}^{\prime}\left(f_{k}\right)$. It means the signal direction $\theta$ and range $d$ should be known previously. In the study, we propose an adaptive far-field transformation approach which utilizes the forward-only adaptive eigen-decomposition (FOAE) algorithm [1] to adjust the signal subspace $E_{s}\left(f_{k}\right)$, and $E_{s}\left(f_{k}\right)$ is transformed to be $E_{s}^{\prime}\left(f_{k}\right)$ by a transformation matrix $Q(\theta(n), d(n)$, $f_{k}$ ) with $\theta(n)$ and range $d(n)$ estimated in the previous iteration.

\section{SIGNAL SUBSPACE FOCUSING (SSF)}

By the proposed AFFT, we have transformed the near-field source estimation to be far-field. Then, in the section, we introduce a signal subspace focusing (SSF) method for wideband source estimation. Our method is based on the transformation of the signal subspace at frequency $f_{k}$ into a focusing subspace at frequency $f_{o}$. The transformation is done by using the focusing matrix, which satisfies

$$
E_{s}^{\prime}\left(f_{o}\right)=T\left(f_{k}\right) E_{s}^{\prime}\left(f_{k}\right)
$$

[4] has shown that a good focusing matrix is unitary. Hence, the focusing matrix in (11) is chosen to be unitary and satisfies the following constrained minimization problem

$$
\min _{T\left(f_{k}\right)}\left\|E_{s}^{\prime}\left(f_{o}\right)-T\left(f_{k}\right) E_{s}^{\prime}\left(f_{k}\right)\right\|_{F}, \quad k=1, \ldots, K
$$

subject to $T^{H}\left(f_{k}\right) T\left(f_{k}\right)=I$. The solution of (12) can be obtained as

$$
T\left(f_{k}\right)=V\left(f_{k}\right) U^{H}\left(f_{k}\right)
$$

In (13), $V\left(f_{k}\right)$ and $U\left(f_{k}\right)$ are the matrices containing the left and right singular vectors of $E_{s}^{\prime}\left(f_{k}\right) E_{s}^{\prime H}\left(f_{o}\right)$, respectively. The focusing matrix in (13) may transform the steering vector $a\left(\theta, f_{k}\right)$ of different frequency bin into the central frequency $f_{o}$,

$$
a\left(\theta, f_{o}\right)=T\left(f_{k}\right) a\left(\theta, f_{k}\right)
$$

Using the far-field transformation matrix $Q\left(\theta_{i}, d_{i}, f_{k}\right)$ and the focusing matrix $T\left(f_{k}\right)$ in (13), the array output vector $\mathbf{X}\left(f_{k}\right)$ is transformed into a new vector,

$$
\mathbf{Z}\left(f_{k}\right)=T\left(f_{k}\right) Q^{H}\left(\theta, d, f_{k}\right) \mathbf{X}\left(f_{k}\right)
$$

Then, we combine the spectrum density matrices of the transformed vector $\mathbf{Z}\left(f_{k}\right)$ over the frequency band by an averaging procedure and construct the smoothed spectrum density matrix

$$
\mathbf{P}_{Z}=\sum_{k} T\left(f_{k}\right) Q^{H}\left(\theta, d, f_{k}\right) \mathbf{P}_{X}\left(f_{k}\right) Q\left(\theta, d, f_{k}\right) T^{H}\left(f_{k}\right)
$$

Using (5), (6) and (14), we get

$$
\mathbf{P}_{Z}=\sum_{i=1}^{q} a\left(\theta_{i}, f_{o}\right) \overline{\mathbf{P}}_{s_{i}} a^{H}\left(\theta_{i}, f_{o}\right)+\sigma^{2} I
$$

where $\overline{\mathbf{P}}_{s_{i}}=\sum_{k=1}^{K} \mathbf{P}_{s_{i}}\left(f_{k}\right)$. Equation (17) shows the smoothed spectrum density matrix $\mathbf{P}_{\mathrm{Z}}$ has the same form as the correlation matrix corresponding to far-field and narrow band sources. If we perform the transformation in (15), the DOA estimation of the near-field wideband sources can be obtained by utilizing any super-resolution signal subspace methods for the DOA estimation of far-field narrowband sources. To estimate the DOA, we average the null spectrum, defined in (10), over frequency band and obtain the smoothed null spectrum which is given by

$$
\bar{p}(\theta)=\frac{1}{K} \sum_{k=1}^{K} p\left(\theta, f_{k}\right)
$$

Substituting (10) into (18) and using (11) and (14), we obtain

$$
\begin{aligned}
\bar{p}(\theta)= & \frac{1}{K} \sum_{k=1}^{K} a^{H}\left(\theta, f_{k}\right) T^{H}\left(f_{k}\right) T\left(f_{k}\right) \\
& {\left[I-E_{s}^{\prime}\left(f_{k}\right) E_{s}^{\prime H}\left(f_{k}\right)\right] T^{H}\left(f_{k}\right) T\left(f_{k}\right) a\left(\theta, f_{k}\right) }
\end{aligned}
$$

$=a^{H}\left(\theta, f_{o}\right)\left[\frac{1}{K} \sum_{k=1}^{K} T\left(f_{k}\right)\left(I-E_{s}^{\prime}\left(f_{k}\right) E_{s}^{\prime H}\left(f_{k}\right)\right) T^{H}\left(f_{k}\right)\right] a\left(\theta, f_{o}\right)$

$=a^{H}\left(\theta, f_{o}\right)\left[I-E_{s}^{\prime}\left(f_{o}\right) E_{s}^{\prime H}\left(f_{o}\right)\right] a\left(\theta, f_{o}\right)$

By searching the smoothed null spectrum, we can estimate the DOAs of wideband sources in near-field.

The proposed adaptive DOA estimator, called adaptive signal subspace and far-field transformation (ASFFT) is summarized as follows:

1) Set the initial DOA and range, $\theta_{i}(0)$ and $d_{i}(0)$, for each source and the initial eigenvectors $E_{s}\left(f_{k}\right)$ for each frequency components.

2) Perform DFT on the array output signals $x_{m}(t)$ for each snapshot and get Fourier components, $X_{m}\left(f_{k}\right)$.

3) Adjust the signal subspace $E_{s}\left(f_{k}\right)$ for each frequency bin from $X_{m}\left(f_{k}\right)$ by the forward-only adaptive eigendecomposition algorithm (FOAE). 
4) Transform the near-field signal subspace $E_{s}\left(f_{k}\right)$ to be farfield signal subspace $E_{s}^{\prime}\left(f_{k}\right)$ for each frequency bin by using the transformation matrix $Q\left(\theta(n), d(n), f_{k}\right)$.

5) Transform the far-field signal subspace $E_{s}^{\prime}\left(f_{k}\right)$ at each frequency bin to the signal subspace at receiver center frequency $E_{s}^{\prime}\left(f_{o}\right)$ by using a focusing matrix $\mathbf{T}\left(f_{k}\right)$.

6) Employ $E_{s}^{\prime}\left(f_{o}\right)$ to do the far-field bearing estimation $\theta(n+1)$ by using root-MUSIC.

7) Adjust the range $d(n+1)$ under MMSE of the smoothed null spectrum by using the gradient searching method.

$$
d(n+1)=d(n)-\mu \bar{p}^{\prime}\left(\theta(n), d(n), f_{o}\right)
$$

where $\mu$ is the step size and $\bar{p}^{\prime}\left(\theta(n), d(n), f_{o}\right)$ is the gradient of smoothed null spectrum which is derived from (19),

$$
\bar{p}^{\prime}\left(\theta(n), d(n), f_{o}\right)=\left.\frac{\partial\left(\bar{p}\left(\theta, d, f_{o}\right)\right.}{\partial(d)}\right|_{\theta=\theta(n), d=d(n)}
$$

In (21), $\bar{p}\left(\theta, d, f_{o}\right)$ defined in (19) is rewritten as

$$
\begin{aligned}
\bar{p} & \left(\theta, d, f_{o}\right) \\
& =\bar{p}(\theta)=a^{H}\left(\theta, f_{o}\right)\left(I-E_{s}^{\prime}\left(f_{o}\right) E_{s}^{\prime H}\left(f_{o}\right)\right) a\left(\theta, f_{o}\right) \\
& =a^{H}\left(\theta, f_{o}, d\right)\left(I-E_{s}\left(f_{o}\right) E_{s}^{H}\left(f_{o}\right)\right) a\left(\theta, f_{o}, d\right)
\end{aligned}
$$

8) Repeat steps 2 to 7 till the gradient of the smoothed null spectrum in (21) approaches zero.

In the future study, the proposed adaptive method will be further modified for coherent sources by combining spatial smoothing and the adaptive eigen-decomposition algorithm.

\section{EXPERIMENT RESULTS}

Simulations are performed to demonstrate the efficiency of the proposed method ASFFT. We conducted experiments based on a linear array of sixteen sensors with equal spacing of half-wavelength at central frequency $f_{o}=100 \mathrm{~Hz}$. The source signals were band-pass zero-mean white Gaussian processes with same central frequency $100 \mathrm{~Hz}$ and band-width $40 \mathrm{~Hz}$. The sampling rate at each array element was chosen to be 160 $\mathrm{Hz}$ and the total observation time was $96 \mathrm{~s}$. We divided the observation time into 120 segments, such that each segment ("snapshot") was $0.8 \mathrm{~s}$. With this sampling scenario, we apply 128-point DFT of the array output signals on each snapshot and get $\mathbf{X}\left(f_{k}\right), k=0, \ldots, 127$. In addition, 33 frequency components $\mathbf{X}\left(f_{k}\right), k=64, \ldots, 96$, corresponding to $80-120 \mathrm{~Hz}$ were selected in the simulation.

We first considered two near-field wideband sources located at direction $\theta_{1}=5^{\circ}$ and $\theta_{2}=10^{\circ}$ and range $d_{1}=15 \lambda$ and

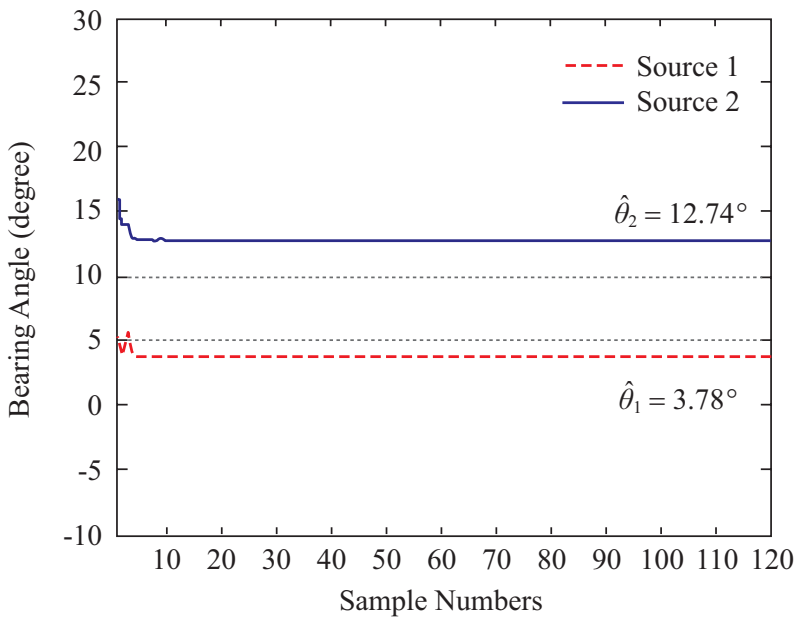

(a) DOA Estimation by ASSF

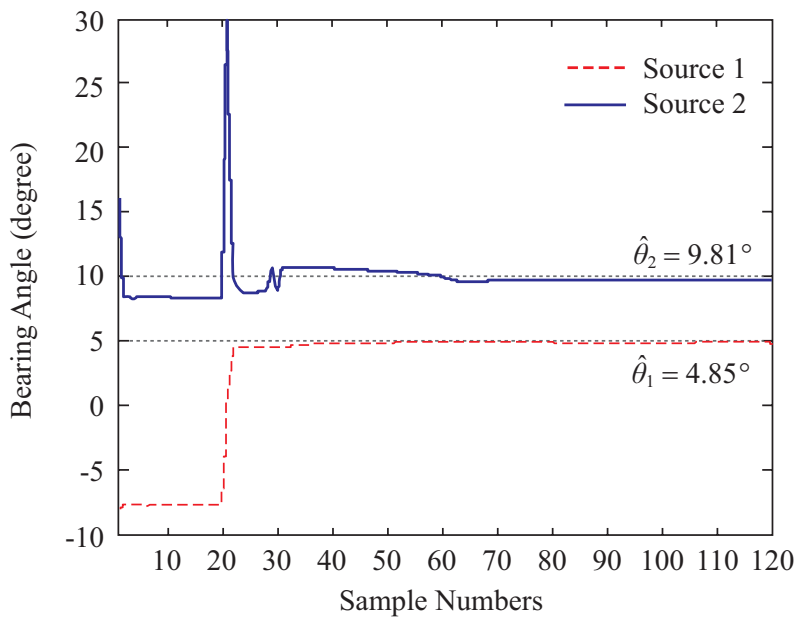

(b) DOA Estimation by ASFFT

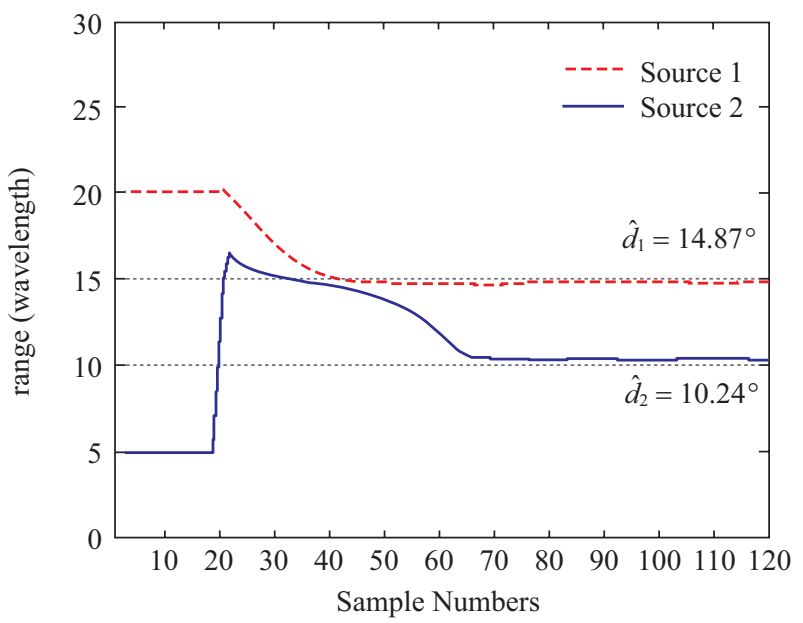

(c) Range Estimation by ASFFT

Fig. 1. The estimation results of two near-field wideband sources located at $\left[\theta_{1}, d_{1}\right]=\left[5^{\circ}, 15 \lambda\right]$ and $\left[\theta_{2}, d_{2}\right]=\left[10^{\circ}, 10 \lambda\right]$.

$d_{2}=10 \lambda$, respectively, with the same $\mathrm{SNR}=10 \mathrm{~dB} . \lambda$ is the wavelength at central frequency, $\lambda=c / f_{o}$. In the simulations, we compare the proposed ASFFT with adaptive signal sub- 


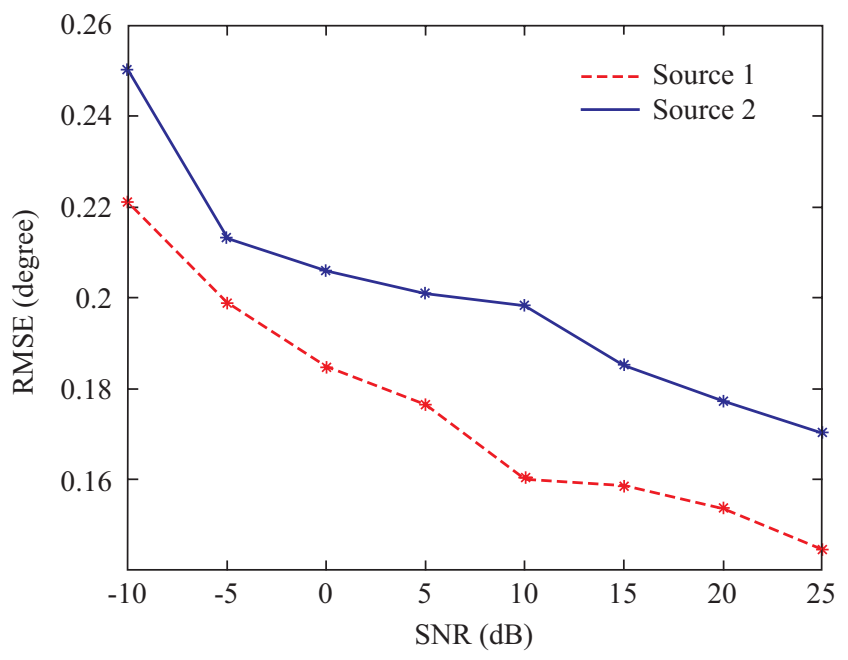

(a) RMSE of the DOA by ASFFT

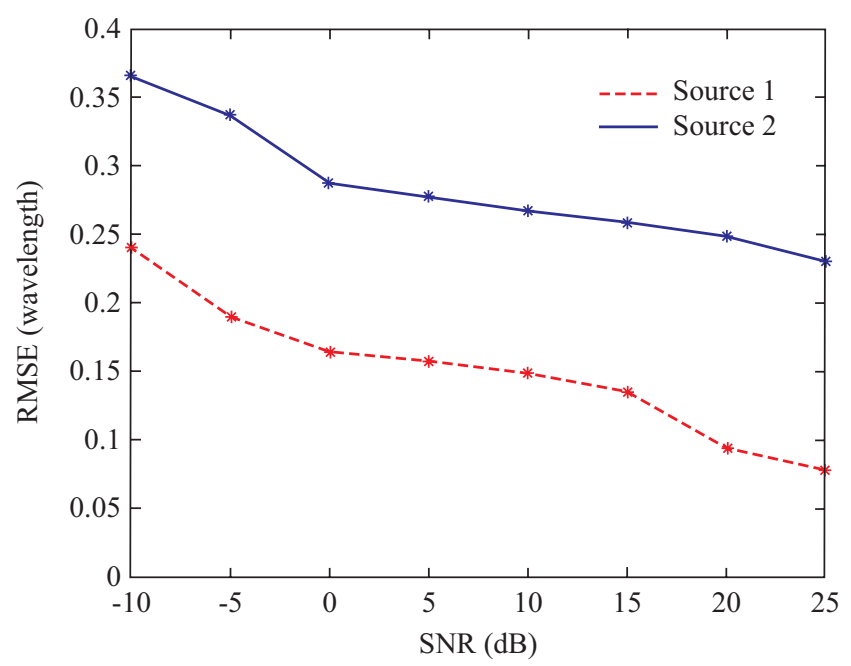

(b) NRMSE of the Range by ASFFT

Fig. 2. Statistics analyses of two near-field wideband sources located at $\left[\theta_{1}, d_{1}\right]=\left[5^{\circ}, 15 \lambda\right]$ and $\left[\theta_{2}, d_{2}\right]=\left[10^{\circ}, 10 \lambda\right]$.

space transformation (ASSF) which combine the the forwardonly adaptive eigen-decomposition (FOAE) algorithm [1] and the signal subspace focusing (SSF) method for far-field wideband source estimation. Furthermore, to validate the efficiency of FFT, we didn't perform FFT, the step 2 of the proposed ASFFT, in the first 20 samples. Figure 1 show the estimated bearings and ranges for 120 samples. The simulation curves were averaged from 100 independent Monte Carlo trials. The results in Fig. 1 indicate that the proposed method can estimate the DOAs more accurate than the ASSF approach. Moreover, we observe that the proposed method can improve the estimation accuracy significantly after 20 samples at which we began performing FFT. The estimated source directions are $\hat{\theta}_{1}=4.85^{\circ}$ and $\hat{\theta}_{2}=9.81^{\circ}$, and rages are $\hat{d}_{1}=14.87 \lambda$ and $\hat{d}_{2}=10.24 \lambda$ after 120 samples. Figure 1 validates that the proposed method can estimate the source directions and ranges simultaneously, with a faster convergence rate and achieves

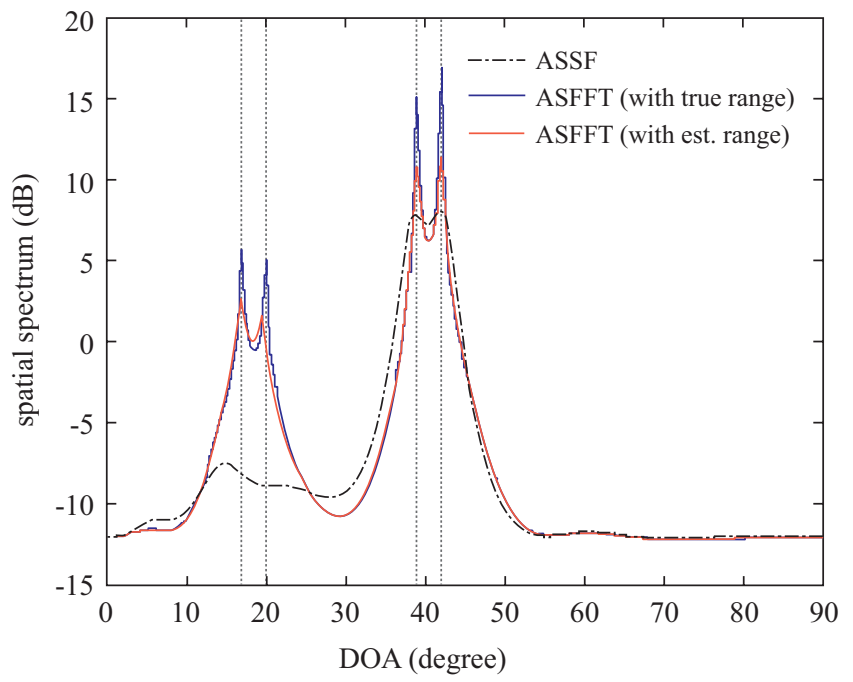

Fig. 3. Inverse of smoothed null spectrum. Two near-field sources located at $\left[\theta_{1}, d_{1}\right]=\left[17^{\circ}, 20 \lambda\right]$ and $\left[\theta_{2}, d_{2}\right]=\left[20^{\circ}, 15 \lambda\right]$, and two far-field sources located at $39^{\circ}$ and $42^{\circ}$, respectively.

high estimation accuracy. We then examine the estimation performance of the proposed ASFFT. The performance is measured by the root mean square error (RMSE) for the DOA and the normalized root mean square error (NRMSE) for the range defined in [2]. The statistics analyses are conducted by 100 independent trials. Figure 2 shows the RMSE for the DOA and RNMSE for the range with SNR varied from $-10 \mathrm{~dB}$ to $25 \mathrm{~dB}$. The RMSE for the DOA and RNMS for the range are smaller than $0.3^{\circ}$ and $0.37 \lambda$, respectively, even for $S N R=-10 \mathrm{~dB}$. In Fig. 2, we observe that the root mean square error for the DOA and range of the source located $\left[\theta_{2}, d_{2}\right]=\left[10^{\circ}, 10 \lambda\right]$ are larger than those of the source located $\left[\theta_{1}, d_{1}\right]=\left[5^{\circ}, 15 \lambda\right]$. The results come from that the near-field effect of source located $\left[\theta_{2}, d_{2}\right]=$ $\left[10^{\circ}, 10 \lambda\right]$ is stronger than the source located $\left[\theta_{1}, d_{1}\right]=\left[5^{\circ}\right.$, $15 \lambda$ ]. From Fig. 2, we also observe that the performance of ASFFT becomes better as SNR increases. The result is reasonable since the estimation of signal subspace is more accurate for larger SNR.

Next, we consider the case of multiple arrivals of which some arrivals locate at near-field and others are at far-field. The impinging four wideband sources are two near-field sources located at $\left[\theta_{1}, d_{1}\right]=\left[17^{\circ}, 20 \lambda\right]$ and $\left[\theta_{2}, d_{2}\right]=\left[20^{\circ}, 15 \lambda\right]$, and two far-field sources located at $\theta_{3}=39^{\circ}$ and $\theta_{4}=42^{\circ}$, respectively. The four sources are with the same $\mathrm{SNR}=10 \mathrm{~dB}$ Figure 3 shows the inverse of smoothed null spectrum in (22), $D\left(\theta, d, f_{o}\right)=1 / \bar{p}\left(\theta, d, f_{o}\right)$ from 100 samples. The peak locations of the spectrum $D\left(\theta, d, f_{o}\right)$ correspond to the DOAs of the arrivals. In the simulations, we compare the estimation property of the proposed ASFFT with estimated ranges $\left[\hat{d}_{1}, \hat{d}_{2}\right]=[20.7 \lambda, 15.9 \lambda]$ and with known true ranges $\left[d_{1}, d_{2}\right]=$ $[20 \lambda, 15 \lambda]$, respectively, to that of the ASSF method. From the spectrum in Fig. 3, we observe that resolution property of the proposed ASFFT is better than the ASSF for two near-field sources. The estimation biases of the ASSF with estimated 


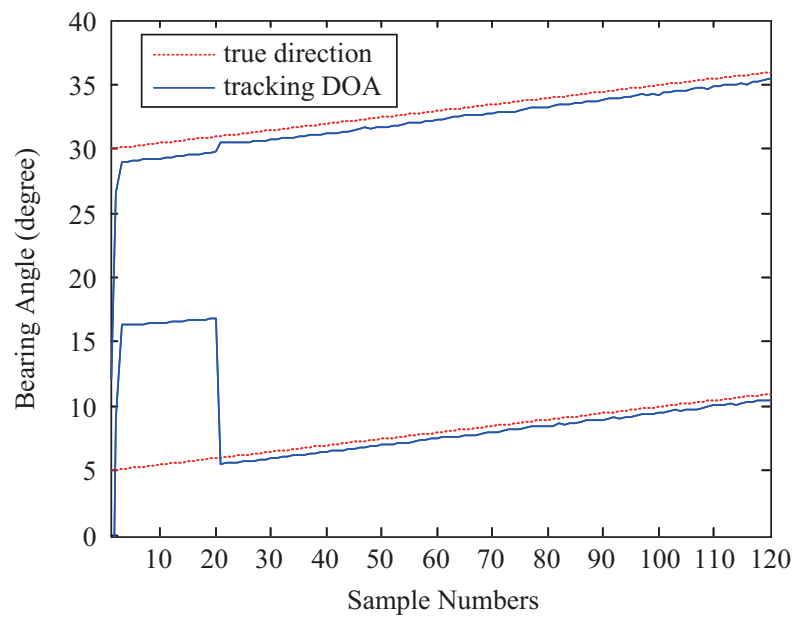

(a) DOA Tracking

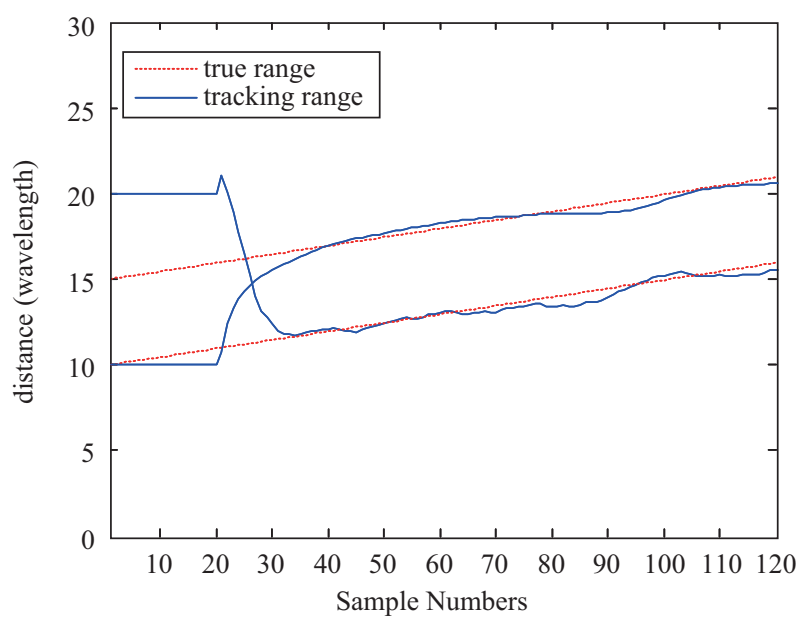

(b) Range Tracking

Fig. 4. The tracking results of two moving near-field wideband sources: $\theta_{1}: 5^{\circ} \rightarrow 11^{\circ}, d_{1}: 10 \lambda \rightarrow 16 \lambda$ and $\theta_{2}: 30^{\circ} \rightarrow 36^{\circ}, d_{2}: 15 \lambda \rightarrow 21 \lambda$.

ranges are $0.1^{\circ}$ and $0.3^{\circ}$ for near-field sources $\theta_{1}=17^{\circ}$ and $\theta_{2}=20^{\circ}$, respectively. Where the ASSF with estimated ranges can estimate the DOAs of far-field sources accurately. The results in Fig. 3 also indicates the proposed ASFFT with known ranges can estimate the DOAs of near-field sources more accurate than ASFFT with estimated ranges.

Then, we examined the tracking capacity by estimating the locations of two moving sources, one moving from $\theta_{1}: 5^{\circ} \rightarrow$ $11^{\circ}$ and $d_{1}: 10 \lambda \rightarrow 16 \lambda$, and the other moving from $\theta_{2}: 30^{\circ} \rightarrow$ $36^{\circ}$ and $d_{2}: 15 \lambda \rightarrow 21 \lambda$. Figure 4 validates that the proposed method is effective in tracking the locations of wideband sources.

Moreover, to examine the robustness of the proposed ASFFT in the multipath environment, we conducted the simulation with sources similar to those in Fig. 3, but the first two signals $s_{1}(t)$ and $s_{2}(t)$ are coherent with $s_{2}(t)=s_{1}(t-0.125 s)$. The results in Fig. 5(a) show that the proposed ASSFT is effective only for non-coherent sources. To overcome the multipath problem, we employ the spatial smoothing technique [6] to decorrelate

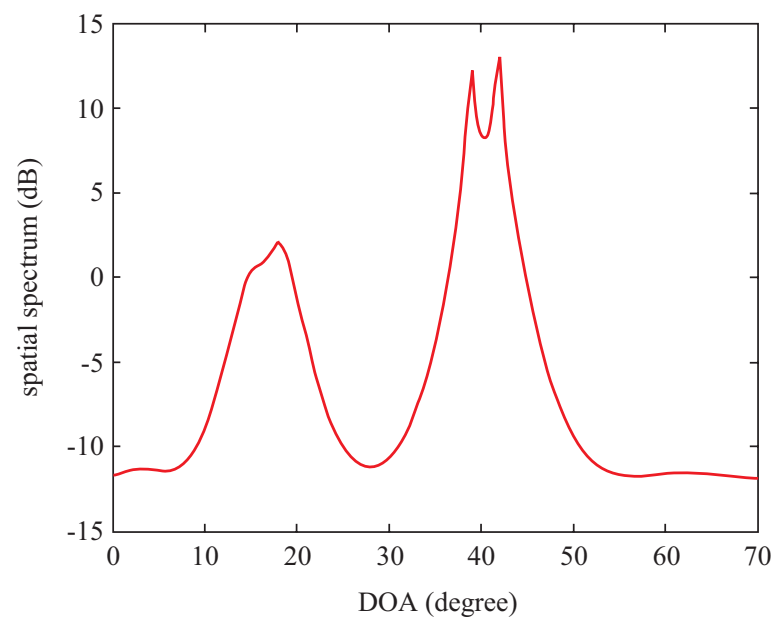

(a) ASSFT

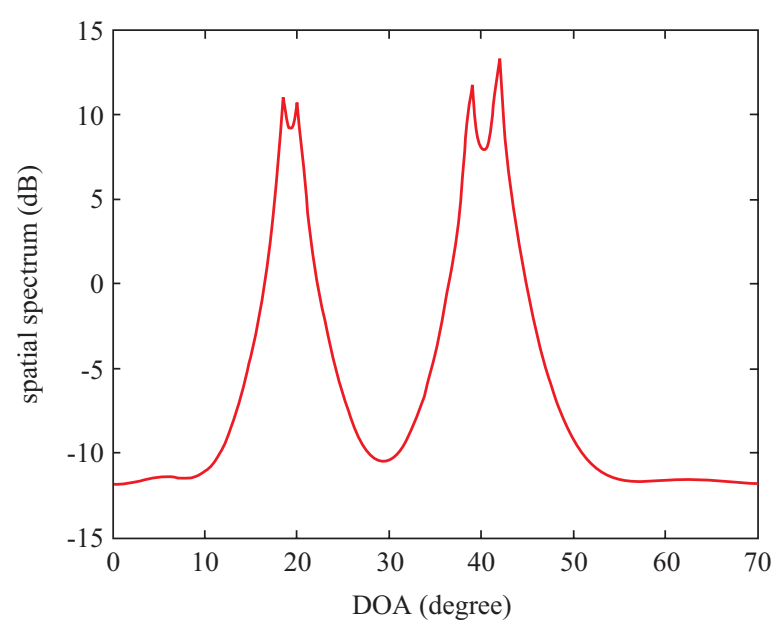

(b) ASSFT with spatial smoothing

Fig. 5. Inverse of smoothed null spectrum. Simulation scenario same as Fig. 3, but two near-field sources are coherent, $s_{2}(t)=s_{1}(t-0.125 s)$.

the coherence of sources. We divided the array into two overlapped subarrays with 15 elements sensors each. The frequency components of each subarray, $\mathbf{X}_{1}\left(f_{k}\right)$ and $\mathbf{X}_{2}\left(f_{k}\right)$ are both used to adjust the signal subspace $E_{s}\left(f_{k}\right)$ in the step 3 of ASSFT. Simulation results in Fig. 5(b) validate the ASSFT combined with the spatial smoothing technique is effective in DOA estimation for coherent wideband sources in near-field.

Finally, we examine the efficiency of the proposed method in underwater environments. The water tank experiment with structure shown in Fig. 6 is performed to simulate the underwater environments. In the experiment, a sine wave with amplitude $\pm 5 \mathrm{~V}$ and radiating frequency $5 \mathrm{~K} \mathrm{~Hz}$ was generated by the signal generator. The transmission hydrophone was located at direction $\theta=10^{\circ}$ and range $d=150 \mathrm{~cm} \approx 5 \lambda$. The bearing estimation results are presented in Fig. 7. We observe that the proposed method converges at around 200 samples. The results indicate the real-time estimation ability of proposed approach. The estimated location is $\hat{\theta}=10.5^{\circ}$ and $\hat{d}=$ $4.9 \lambda$ at 500 samples. The estimation bias may be caused by 


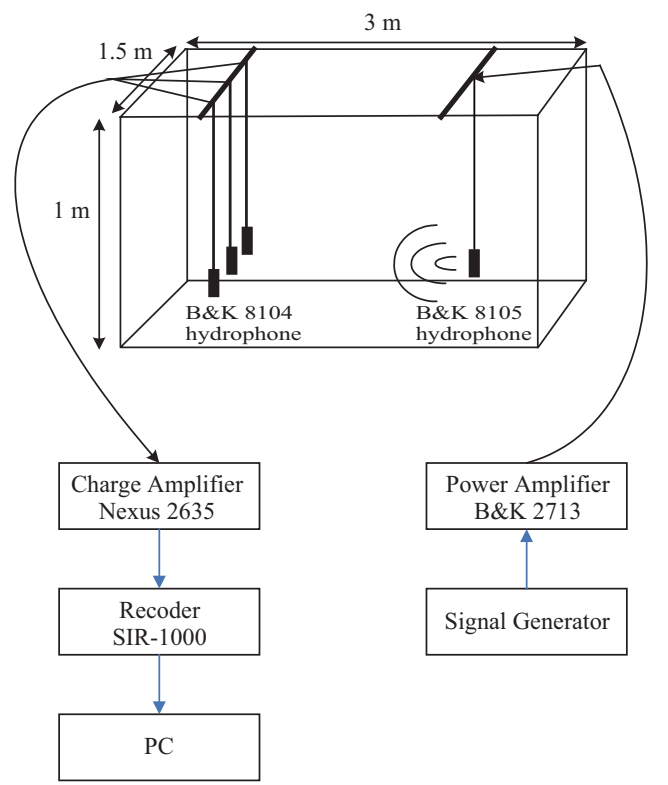

Fig. 6. Block diagram of water tank experiment.

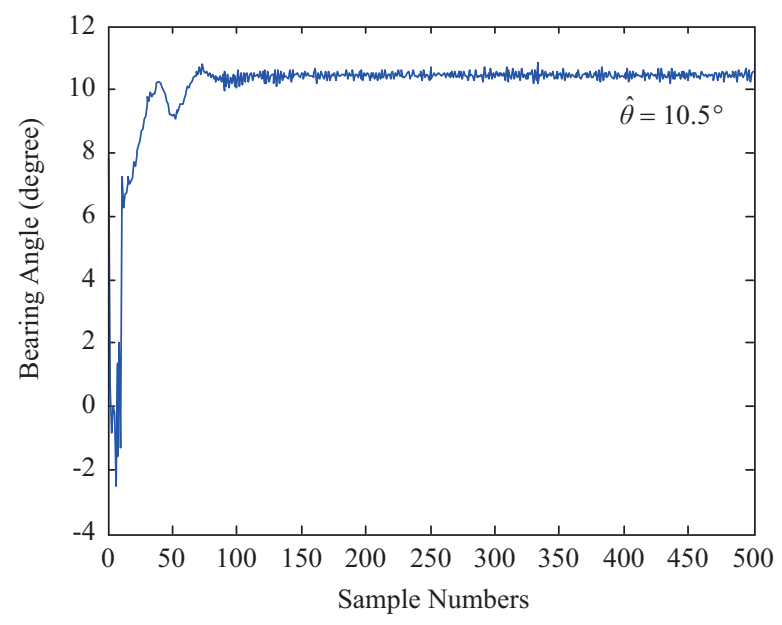

(a) DOA Estimation

Fig. 7. The estimation results of a source with radiation frequency 5 $\mathrm{KHz}$, located at $\theta=10^{\circ}$ and $d=5 \lambda$, in underwater environments. the reflections from water tank and the small size of receiving antenna (with 3 sensors in the experiment). In the future study, we will improve the estimation accuracy by using a larger antenna and do experiments in real underwater environments.

\section{CONCLUSIONS}

In this paper, we proposed the ASFFT method for DOA and range estimations of wideband sources in the near-field. The ASFFT combines SSF and FFT. SSF transforms the signal subspace corresponding to each frequency bin on to the focusing subspace by using a focusing matrix. FFT transforms the near-field steering vector to be the far-field steering vector with the same DOA. By performing the SSF and FFT adaptively, we simplify the location estimation of wideband sources in the near-field to be narrowband sources in the far field. Simulations demonstrate the estimation accuracy and track efficiency of the proposed ASFFT which can estimate the DOAs and ranges of near-field wideband sources, simultaneously. And the water-tank experiments confirm the feasibility and efficiency of the proposed ASFFT method in underwater environments.

\section{REFERENCES}

1. Chang, L., "Adaptive eigendecomposition algorithms for eigenstructurebased array signal processing," Journal of the Chinese Institute of Engineers, Vol. 20, No. 4, pp. 365-375 (1997).

2. Grosicki, E., Abed, M. K., and Hua, Y., "A weighted linear prediction method for near-field source localization," IEEE Transactions on Signal Processing, Vol. 53, pp. 3651-3660 (2005).

3. Huang, Y. D. and Barkat, M., "Near-field multipe source localization by passive sensor array," IEEE Transactions on Antennas and Propagation, Vol. 39, No. 7, pp. 968-975 (1991).

4. Hung, H. and Kaveh, M., "Focusing matrices for coherent signalsubspace processing," IEEE Transactions on Acoustics, Speech, Signal Processing, Vol. 36, pp. 1272-1281 (1988).

5. Lee, J. H., Chen, Y. M., and Yeh, C. C., "A covariance approximation method for near-field direction-finding using a uniform linear array," IEEE Transactions on Signal Processing, Vol. 43, No. 5, pp. 1293-1298 (1995).

6. Shan, T. J., Wax, M., and Kailath, T., "On spatial Smoothing for directionof-arrival estimation of coherent signals," IEEE Transactions on Acoustics, Speech, Signal Processing, Vol. 33, No. 4, pp. 806-811 (1985).

7. Sun, D. J. and Zeng, W. J., " Non-iterative deconvolution algorithm applied to shallow water multipath channel estimation in near range," Journal of Harbin Engineering University, Vol. 25 , No. 1, pp. 30-33 (2004).

8. Tichavský, P., Wong, K. T., and Zoltowski, M. D., "Near-field/far-field azimuth and elevation angle estimation using a single vector hydrophone," IEEE Transactions on Signal Processing, Vol. 49, No. 11, pp. 2498-2510 (2001).

9. Valaee, S. and Kabal, P., "Estimation of directions of arrival of wideband and wideband spread sources" Signal Processing, Vol. 87, pp. 614-622 (2007).

10. Yoon, Y. S., Kaplan, L. M., and McClellan, J. H., “TOPS: New DOA estimator for wideband signals," IEEE Transactions on Signal Processing, Vol. 54, pp. 1977-1989 (2006).

11. Zeng, W. J., Li, X. L., Zou, H., and Zhang, X. D., "Near-field multiple source localization using joint diagonalization," Signal Processing, Vol. 89, No. 2, pp. 232-238 (2009).

12. Zhi, W. and Chia, M. Y-W., "Near-field source location via symmetric subarrays," IEEE Signal Processing Letters, Vol. 14, pp. 409-412 (2007). 